\title{
Application of Dried Egg White to Enhance the Textural Properties of Fresh and Frozen Pancakes
}

Emerson Nolasco, ${ }^{1}$ Emily Jundt, ${ }^{1}$ Madeline M. Kramp, ${ }^{1}$ Enakshy Dutta, ${ }^{2}$ Noriaki Nishijima, ${ }^{3,4}$ Yoshifumi Oda, ${ }^{3,4}$ Akihiro Handa, ${ }^{3}$ Changmou Xu, ${ }^{1}$ Yue Zhang, ${ }^{1,5}$ and Kaustav Majumder ${ }^{1, *}$

\begin{abstract}
The bakery industry trends such as sugar reduction have encounter challenges as undesirable texture properties. Polysaccharides and proteins can enhance the physical and textural properties of fresh and frozen bakery products. Additionally, egg white proteins possess superior foaming and emulsifying properties. Thus, there is a critical need to identify if pancake's physical and texture properties benefit from the functional properties of egg white. Egg white powder solids M200 and P110 were incorporated in formulations with and without whole egg at $1 \%$ and $3 \%$ level. Thickness, hardness, gumminess, chewiness, and pore size elucidated the effect of M200 and P100 in fresh and frozen pancakes. Here we show that egg white powder solids M200 improve the fresh pancake's textural properties while maintaining its thickness after freezing. Formulations with and without whole egg, including M200, showed a higher thickness, lower hardness and gumminess, and a reduced chewiness. Pancake formulations containing whole egg and M200 developed filaments in the edge of the pores, reinforcing its network. Our results provide insight into the functional properties of egg white protein, M200 for preparing fresh and frozen pancakes, which could further improve consumer acceptability and provide a solution to reduced-sugar bakery texture limitations.
\end{abstract}

Keywords: Egg white powder; Scanning Electron Microscope; Foaming; Texture Properties; Frozen Foods.

Received: 23 November 2020; Accepted: 27 December 2020.

Article type: Research article.

\section{Introduction}

Egg white is commonly used in baked goods for their structural and foaming properties. ${ }^{[1]}$ It acts as a binder in the product $^{[2]}$, providing volume and aeration. Whole fresh eggs are fragile, difficult to transport, and non-shelf stable. ${ }^{[3]}$ As a result, there is a demand for dried egg products in the food industry because of their extended shelf life and safety ${ }^{[4]}$ Additionally, it can maintain or may enhance the functional properties of a fresh egg after proper processing. ${ }^{[3]}$ Using egg

${ }^{1}$ Department of Food Science and Technology, University of NebraskaLincoln, Lincoln, NE, 68588-6205, United States

${ }^{2}$ Department of Statistics, University of Nebraska-Lincoln, Lincoln, NE, 68583-0963, United States

${ }^{3}$ Institute of Technology Solution, R\&D Division, Kewpie Corporation,

2-5-7 Sengawa, Chofu, Tokyo, 1820002 Japan

${ }^{4}$ Henningsen Foods, Inc., Omaha, NE, 68144, United States

${ }^{5}$ School of Food Science and Biotechnology, Zhejiang Gongshang

University, Hangzhou, 310018, China.

*E-mail: kaustav.majumder@unl.edu (K. Majumder) white powder is beneficial for baked goods due to its foaming properties, taking advantage of it without adding any additional lipids or cholesterol ${ }^{[5]}$ Therefore, egg powders can be incorporated into many different types of foods that need to remain shelf-stable.

Pancakes, a bakery product, is an everyday breakfast staple in the United States and other countries such as Canada, France, Japan, and Korea. ${ }^{[6,7]}$ Pancakes are often bought as a box mix, water-only mix, or frozen pancakes and widely used by restaurants, food services, and individual consumers for domestic uses. ${ }^{[8]}$ Soft wheat flour used for pancake production is often chlorinated to increase volume and improve crumb texture. However, gluten loses part of its network forming ability ${ }^{[9]}$, while the starch in the mix remains responsible for the structure formation. ${ }^{[10]}$ The starch network represents a limitation for frozen bakery products such as cakes, as freezing and thawing results in an increase in firmness due to dense structure, lower moisture content, and starch retrogradation. ${ }^{[11]}$

Egg white powder has shown to improve the texture 
properties of frozen pasta. ${ }^{[12]}$ A freeze-thaw process on egg white can increase its foaming properties derived from an increased exposure of egg white proteins hydrophobic groups, enhancing its air-water interface interaction. ${ }^{[13]}$ Despite egg whites promising characteristics in pasta products and freezing conditions, its impact on pancakes remains unidentified. Thus, this study utilizes two forms of egg white powder and aims to determine their effect at low concentrations on fresh and frozen pancakes. The study objective was to evaluate the impact of incorporating dried egg white powder solids (EWPS) at low levels on the textural and physical properties of fresh and frozen pancakes.

\section{Materials and Methods}

\subsection{Materials}

Sprayed dried egg white powder solids (EWPS) M200 and P110 were provided by Henningsen Foods, Inc. (Omaha, NE). Pancake mix, whole milk, whole eggs, soy protein, and shortening were acquired from the local market. Two pancake treatments were devised for this study: using the whole egg (WE) and EWPS (treatment 1(T1)) and using EWPS only (treatment 2 (T2)). The positive control (C1) for T1 was prepared according to a commercial pancake mix, requiring milk and whole egg. The negative control (C2) for T2 used soy protein as a replacement for the egg. Water and shortening were used to substitute whole egg moisture and lipids. Neither of the controls contained EWPS. The commercial treatment for fresh pancakes constituted water only pancake mix and for reheated pancakes a commercial frozen pancake product. The formulations are shown in Table 1.

\subsection{Preparation of pancakes}

Pancakes were prepared in $300 \mathrm{~g}$ batches, which yielded 6-7 pancakes. After being weighed, the dry ingredients were homogenized in a Kitchen-Aid Professional Stand Mixer (KitchenAid, St. Joseph, MI), fitted with a paddle attachment, for $30 \mathrm{~s}$ on stir speed (Scale $=1-10)$. The wet ingredients were added and homogenized for an additional $30 \mathrm{~s}$ on stir speed (for $\mathrm{T} 2$, the shortening was melted before incorporation). The sides of the bowl were scraped, and the batter was mixed on speed 2 for $30 \mathrm{~s}$. A nonstick electric griddle was preheated to $176^{\circ} \mathrm{C}\left(350^{\circ} \mathrm{F}\right)$. A metal cooking scoop $(2.8 \mathrm{~cm}$ height) was used to portion the batter, which resulted in a crude weight of 31-35 g. One scoop of batter was cooked at a time for $90 \mathrm{~s}$ on each side. The pancake was cooled on a rack for $20 \mathrm{~min}$ before being analyzed. Three pancakes from each batch were separated by parchment paper, placed in plastic bags, and frozen for 14 days before analysis. They were defrosted by stacking three pancakes and microwaving on high for $70 \mathrm{~s}$ or $45 \mathrm{~s}$ for two pancakes. The defrosting time was based on the instructions for commercially frozen pancakes.

\subsection{Pancake thickness}

The pancake thickness (in $\mathrm{cm}$ ) was measured using a Vernier caliper. Four measurements were taken at different sides to calculate the average thickness. All the fresh measurements were taken in triplicate and frozen in duplicate.

\subsection{Texture Profile Analysis}

A TA.TX2 Texture Analyzer (Stable Micro Systems Ltd., Godalming, UK) coupled with a 3" diameter plunger performed the texture profile analysis (TPA) in fresh and reheated pancakes to determine the hardness, gumminess, and chewiness of the product. The test speed was $1 \mathrm{~mm} / \mathrm{s}$, with a strain target mode of $50 \%$. The time set was $5 \mathrm{~s}$ with an autotrigger force of $5 \mathrm{~g}$.

\subsection{Scanning Electron Microscopy}

Frozen pancakes after one day of storage at $-80{ }^{\circ} \mathrm{C}$ were freeze-dried. Afterward, sections of $1 \times 1 \times 1 \mathrm{~cm}$ were prepared. The samples were dried in a vacuum oven at $41{ }^{\circ} \mathrm{C}$ overnight. The dried pancake sections were fixed on aluminum SEM stubs and sputter coated. The pore size was observed by imaging using a Hitachi S-4700 field-emission scanning electron microscopy (SEM) with $10.0 \mathrm{kV}$ and a $30 \mathrm{x}$ magnification. The network structure was observed with a 500x magnification. The images were taken on duplicate samples.

Table 1. Pancake formulas with and without egg white solids.

\begin{tabular}{|c|c|c|c|c|c|c|c|c|c|c|}
\hline Formulas & $\mathrm{C} 1$ & $\mathrm{C} 2$ & WE-P1\% & WE-P3\% & $\begin{array}{l}\text { WE- } \\
\text { M1\% }\end{array}$ & $\begin{array}{l}\text { WE- } \\
\text { M3\% }\end{array}$ & $\mathrm{P} 1 \%$ & $\mathrm{P} 3 \%$ & M1\% & M3\% \\
\hline Ingredients & (g) & & & & & & & & & \\
\hline Pancake Mix & 123 & 123 & 123 & 123 & 123 & 123 & 123 & 123 & 123 & 123 \\
\hline Milk & 149 & 149 & 149 & 149 & 149 & 149 & 149 & 149 & 149 & 149 \\
\hline Whole egg & 28 & - & 28 & 28 & 28 & 28 & - & - & - & - \\
\hline Egg white & - & - & 3 & 9 & 3 & 9 & 3 & 9 & 3 & 9 \\
\hline Water & - & 21 & - & - & - & - & 21 & 21 & 21 & 21 \\
\hline Soy Protein & - & 6 & - & - & - & - & - & - & - & - \\
\hline Shortening & - & 2 & - & - & - & - & 2 & 2 & 2 & 2 \\
\hline Total & 300 & 301 & 303 & 309 & 303 & 309 & 298 & 304 & 298 & 304 \\
\hline
\end{tabular}

Note: C1: Positive Control, C2: Negative Control, WE-P1\%: whole egg + 1\% P110 egg white powder, WE-M1\%: whole egg + 1\% M200 egg white powder, P1\%: No whole egg + 1\% P110 egg white powder. The same rule applies for WE-P3\%, WE-M3\%, P3\%, M1\%, and M3\%. Water and shortening were used to substitute whole egg moisture. 


\subsection{Pore Size Analysis}

The 30x magnification SEM images were incorporated into Matlab (MathWorks, MA) and analyzed through the auto clustering option in the image segmenter application. The data was segmented, and the pore size taken into consideration were from $1 \mathrm{~mm}$ to $8 \mathrm{~mm}$ in diameter. A pore size of less than $1 \mathrm{~mm}$ was considered as noise.

\subsection{Statistical analysis}

The statistical analysis was performed through a one-way ANOVA comparison between treatments to include the controls and commercial pancakes along with pancake treatments with different EWPS percentages. Significant differences were determined using Tukey's multiple comparisons post-hoc test at the level of $P<0.05$. The pore size analysis data was transformed through a square root function to ensure normality. Afterward, a non-parametric one-way ANOVA was used to determine any differences between treatments by pore size using Dunn's multiple comparisons test at the level of $P<0.05$. The data were expressed as mean \pm standard deviation (SD). Measurements were taken in triplicate for fresh pancakes and duplicate for frozen pancakes regarding physical and texture analysis. The statistical analysis was realized with GraphPad Prism software version 8.0.1 (San Diego, CA, USA).

\section{Results}

3.1. Thickness improvement in fresh and frozen pancake by egg white powder solids

One of the main attributes improved by the addition of M200 EWPS was thickness. In T1, at $1 \%$ concentration (Fig. 1A), M200 produced a significantly thicker product than the $\mathrm{C} 1$ and P110 at the same concentration $(P<0.05)$. The frozen and reheated pancakes with $1 \%$ EWPS and $\mathrm{C} 1$ were significantly thicker than a commercially frozen pancake after defrosting (Fig. 1B). At a 3\% concentration, M200 produced a significantly thicker pancake than $\mathrm{C} 1$, commercial, and $3 \%$ P110 (Fig. 1C). After reheating, pancakes from T1 with 3\% EWPS were thicker than $\mathrm{C} 1$ and the commercial frozen pancake (Fig. 1D). In the absence of the whole egg (T2), no significant difference in thickness was found for fresh and reheated pancakes with $1 \%$ EWPS (Fig. S1). However, fresh pancakes containing both EWPS at 3\% were significantly thicker than the commercial and $\mathrm{C} 2$ pancakes but not to each other (Fig. 1E). Finally, C2 and pancakes with EWPS remained thicker than the commercial treatment after reheating (Fig. 1F). M200 was thicker than $\mathrm{C} 2$ and commercial treatment. P110 differed from the commercial treatment but not from the control $(P=0.0618)$.
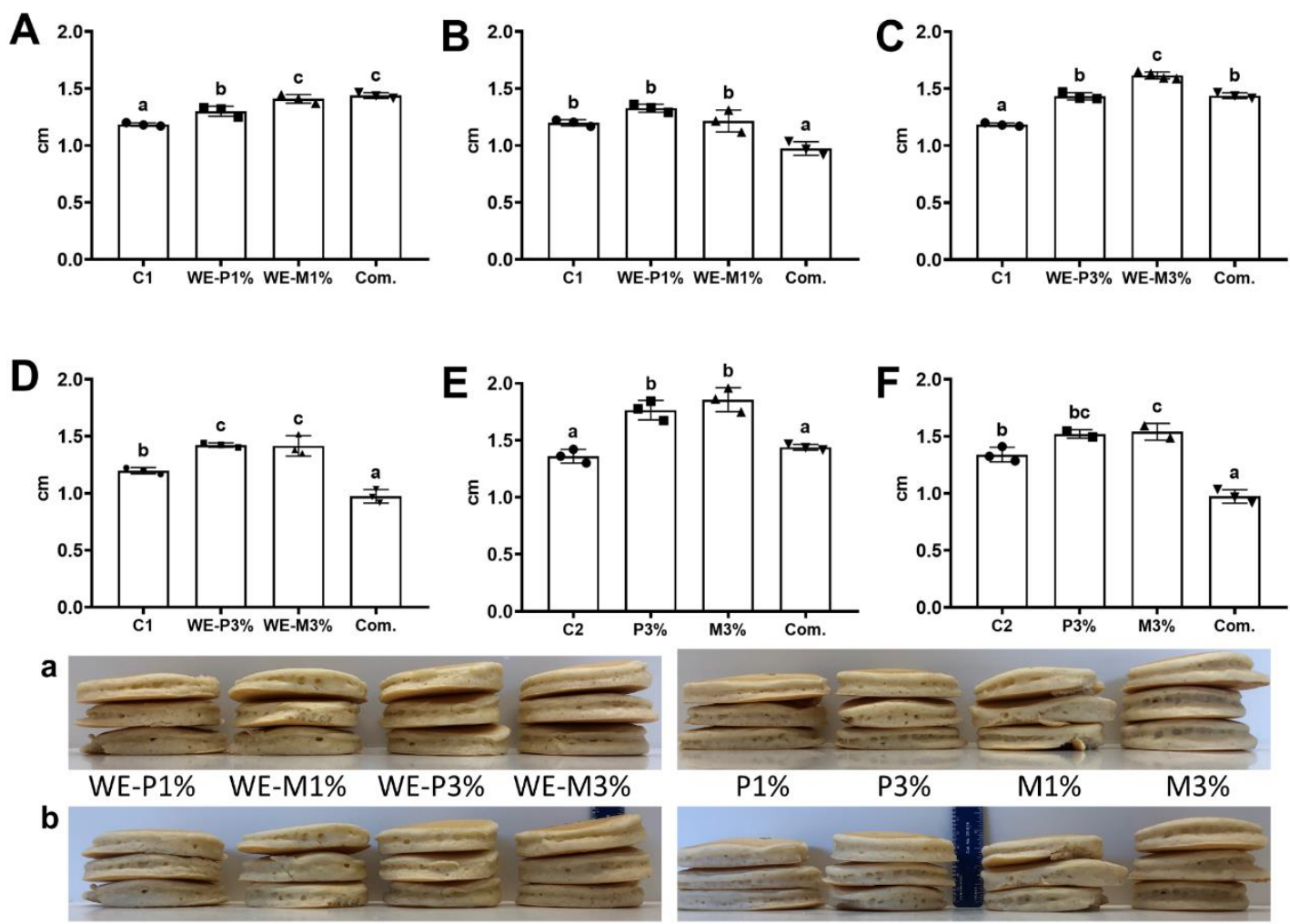

Fig. 1 Thickness of fresh and reheated pancake with egg white powder solids (EWPS) in combination with and without whole egg. Note: A: Treatment (T) $1+1 \%$ egg white powder solids (EWPS), B: T1 reheated + 1\% EWPS, C: T1 + 3\% EWPS, D: T1 reheated $+3 \%$ EWPS, E: T2 + 3\% EWPS, F: T2 reheated + 3\% EWPS. a row: fresh and b row: frozen pancakes. Numbers are mean \pm standard deviation (fresh: $n=3$, reheated: $n=2$, control and commercial reheated: $n=3$ ). Mean values with a different letter between columns indicate a statistical difference $(P<0.05)$. 
Egg white powder functional properties like emulsification and foaming are of benefit in bakery products. ${ }^{[14-16]}$ The higher thickness of pancakes containing EWPS could be the result of EWPS foaming capacity, influenced by the hydrophobic nature of the protein and the surface tension reduction. The surface properties of both EWPS were studied by Han et al. $2019 .{ }^{[17]}$ At $1 \%$ protein concentration in water, M200 showed a faster rate of surface tension decay and reached the equilibrium, the steady state surface tension value faster compared to P110. The study demonstrated the ability of M200 in developing a more stable gluten-free network with a higher capacity to entrap more air than sample P110. This could be the reason here, leading to a thicker product achieved by M200. However, the behavior of egg white protein combined with whole egg together on the physical attributes of the pancake is unknown.

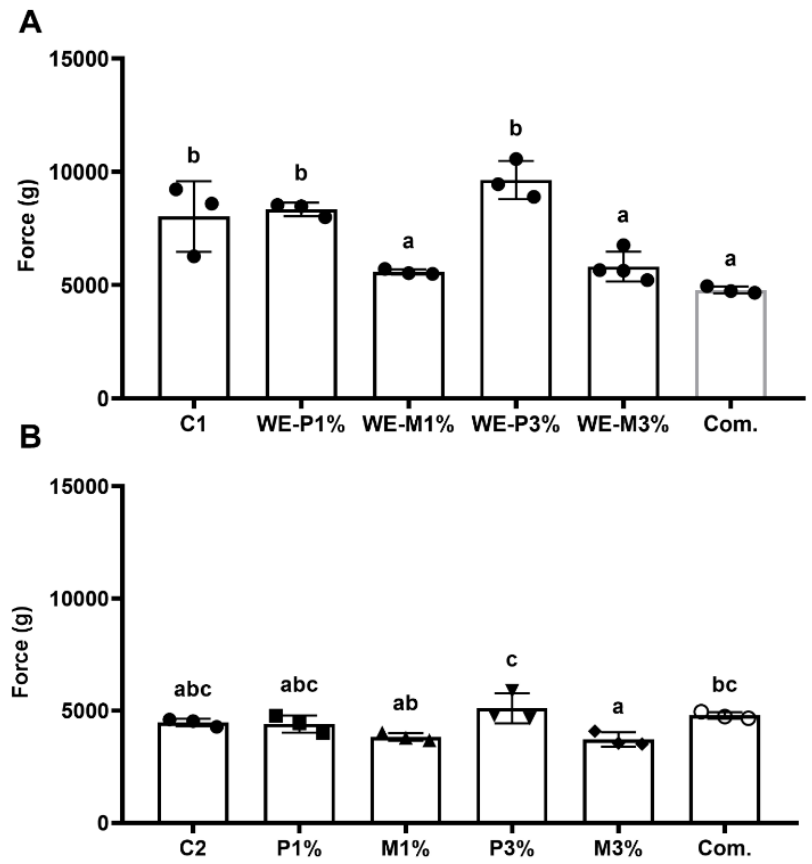

Fig. 2 Hardness of fresh pancake with egg white powder solids (EWPS) in combination with and without whole egg. Note: A: Treatment (T) $1+1 \%$ and $3 \%$ EWPS, B: T2 $+1 \%$ and $3 \%$ EWPS. Numbers are mean \pm standard deviation (fresh: $n=3$ ). Mean values with a different letter between columns indicate a statistical difference $(P<0.05)$.

3.2. Impact of egg white powder in fresh pancake hardness In T1, the use of EWPS in pancakes had an impact on the hardness of fresh pancakes. At $1 \%$ and 3\% concentration, M200 produced a much softer pancake than P110 and C1 (Fig. 2A). In T2, M200 1\% hardness ( $3830 \mathrm{~g}$ ) was not different from the negative control (4471 g), the commercial pancake (4786 g), and P110 1\% (4404 g) (Fig. 2B). When increasing the concentration, M200 at $3 \%$ was softer $(3717 \mathrm{~g})$ than P110 at $3 \%(5110 \mathrm{~g})$ and the commercial pancake (4786 g), with no difference with the negative control (Fig. 2B). The freezing and reheating process eliminated the significant differences in hardness at both concentrations (Fig. S2). As per Fig. 1, Fig. 2, Fig. S1, and Fig. S2, these results suggest a higher thickness can be achieved by 3\% M200 in the presence of whole egg without affecting the hardness of the fresh pancakes.

The low hardness derive by M200 incorporation has also been observed in pasta products. ${ }^{[12]}$ When incorporated in pasta at $3 \mathrm{~g}$ and $5 \mathrm{~g} / 100 \mathrm{~g}$ semolina flour, pasta containing M200 had a significantly higher water absorption and lower cooking loss compared to P110. This was related to the pasta hardness in which M200 at $3 \mathrm{~g} / 100 \mathrm{~g}$ showed a lower hardness compared to P110 in fresh and reheated pasta. The high hardness of pasta with P110 was partly attributed to its low water absorption. ${ }^{[12]}$ The increase in hardness relative to $\mathrm{C} 2$ and derived from an increase of EWPS in T2 was previously observed in bakery products such as gluten-free bread. ${ }^{[18]} \mathrm{An}$ addition of $2 \%$ soy protein isolate and $5 \%$ egg white solids did not affect the bread hardness as $10 \%$ and $15 \%$ egg white solids. This increase was attributed to egg white gelling properties. ${ }^{[18]}$ Therefore, the gel characteristics from the incorporated product are essential to avoid a negative impact on the hardness.

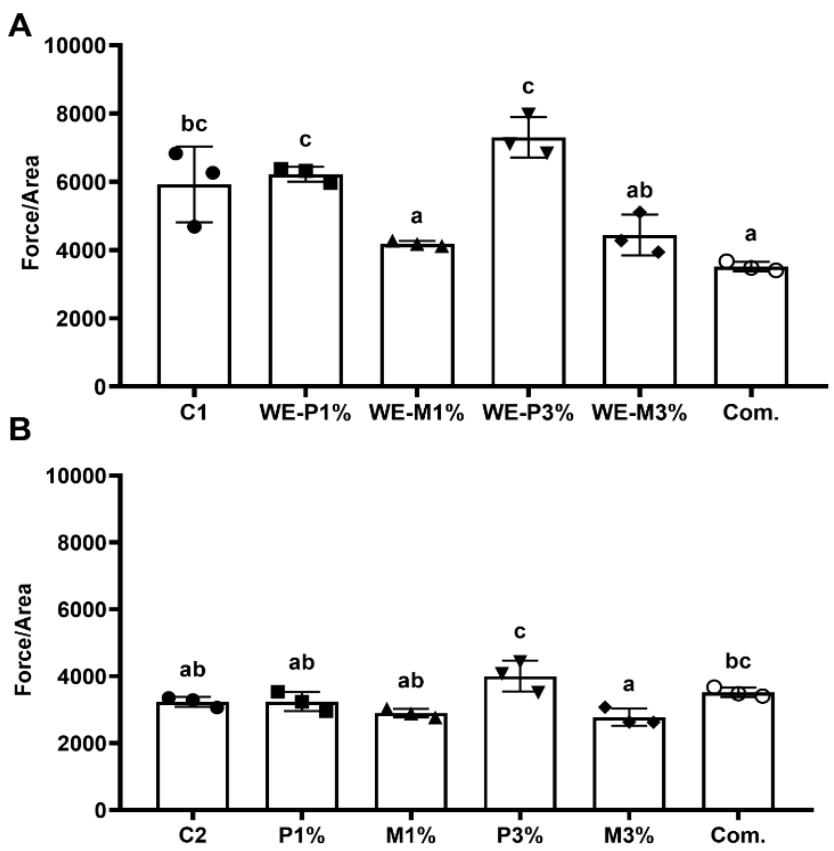

Fig. 3 Gumminess of fresh pancake with egg white powder solids (EWPS) in combination with and without whole egg. Note: A: Treatment (T) $1+1 \%$ EWPS, B: T1+3\% EWPS, C: T1 $1 \%$ and $3 \%$ EWPS, D: T2 $+1 \%$ and $3 \%$ EWPS. Numbers are mean \pm standard deviation (fresh: $n=3$ ). Mean values with a different letter between columns indicate a statistical difference $(P<0.05)$.

\subsection{Effect of egg white powder on fresh pancake gumminess}

The TPA also measured the gumminess; a lower value is favorable for a pancake. In T1 fresh pancakes, M200 at 1\% concentration produced a less gummy pancake than P110 1\%, 
$\mathrm{C} 1$, and P110 at 3\% concentration (Fig. 3A). Overall, M200 lowered gumminess compared to P110 in fresh pancakes (Fig. 3A). After freezing, no significant differences were found between both EWPS and compared to $\mathrm{C} 1$ and the commercial treatment (Fig. S3). The T2 fresh pancakes with M200 at 1\% and 3\% were the least gummy when compared to P110 3\%, while no significant difference was observed for P110 $1 \%$ and the negative control. After freezing, the $\mathrm{C} 2$ and EWPS showed a lower gumminess compared to the commercial pancake only (Fig. S3).

Gumminess is the energy required for disintegrating a semi-solid food to a condition of swallowing and is influenced by hardness as its determination is based on the hardness $x$ cohesiveness values. ${ }^{[19]}$ Incorporation of gums such as crude Malva nut gum, significantly decreased the hardness and gumminess when included in a wheat flour paste $(5-10 \%)$ comparable to gum Arabic. ${ }^{[20]}$ In contrast, guar gum and locust bean gum increased hardness ${ }^{[20,21]}$ Gum Arabic solutions (6\%) develop weak gels characteristics on standing for $2 \mathrm{~h}$, attributed to intermolecular aggregation. ${ }^{[22]}$ Similar to crude Malva nut gum and gum Arabic, M200 could decrease the hardness and gumminess in pancakes due to its water holding capacity, superior foaming properties, and gel characteristic when incorporated in a complex food system. ${ }^{[12,17]}$
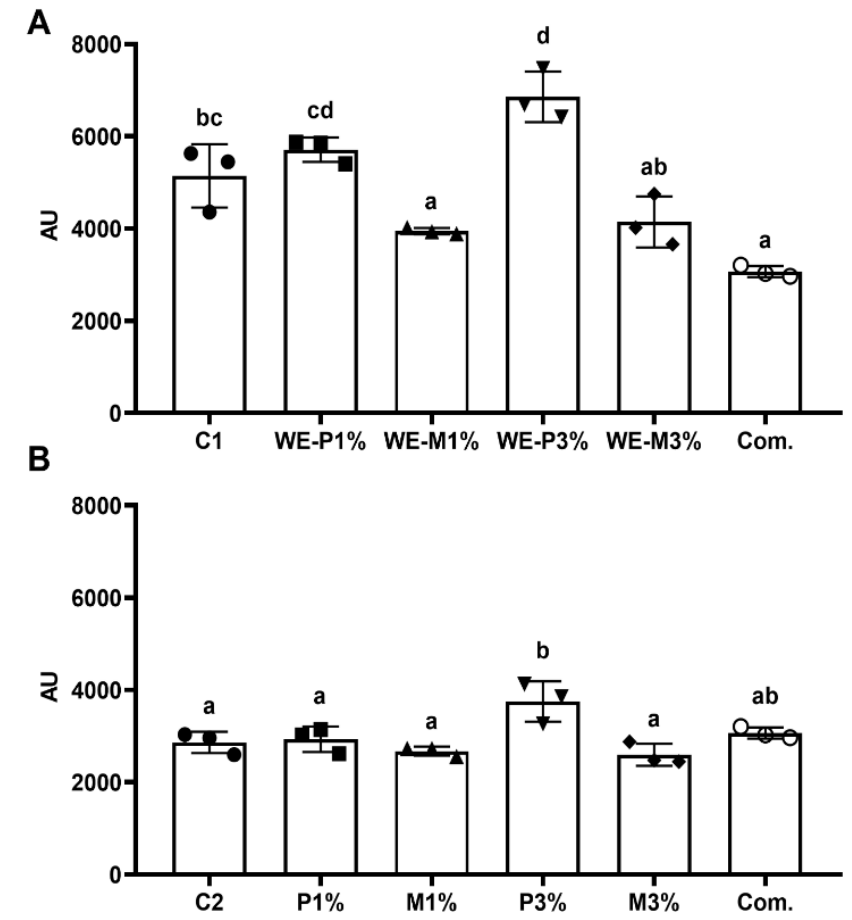

Fig. 4 Chewiness of reheated pancakes with egg white powder solids (EWPS) in combination with and without whole egg. Note: A: Treatment (T) 1 reheated $+1 \%$ and $3 \%$ EWPS, B: T2 reheated $+1 \%$ and $3 \%$ EWPS. Numbers are mean \pm standard deviation (fresh: $\mathrm{n}=3$ ). Mean values with a different letter between columns indicate a statistical difference $(P<0.05)$.

3.4 Effect of egg white powder on fresh pancakes chewiness M200 had a positive impact on the chewiness of pancakes. At both concentrations in T1, M200 decreased the chewiness in fresh pancakes compared to P110 (Fig. 4A). When compared to $\mathrm{C} 1$ and $\mathrm{P} 1101 \%$, the chewiness was reduced by M200 at $1 \%$ (Fig. 4A). The chewiness increased for pancakes with EWPS at $3 \%$ in which M200 did not differ from C1, while P110 3\% did have a significantly higher chewiness. In T2, M200 1\% chewiness did not differ when compared to P110 $1 \%$ (Fig. 4B). When comparing the EWPS at both concentrations, we observed the chewiness is higher for P110 at 3\%, while 1\% P110 and M200 at both levels had a significantly lower chewiness (Fig. 4B). Furthermore, the negative control and EWPS pancakes subjected to freezing and reheating in $\mathrm{T} 2$ had a lower chewiness than the commercial sample, but no significant difference was found between them (Fig. S4).

The chewiness is the energy required for masticating a semi-solid food to a steady state of swallowing ${ }^{[23]}$ and is also dependent on food composition. In rice, a combination of gelling compounds with a specific type of rice starch yield a different response on the chewiness. While Indica rice starchpolysaccharide gel had a high chewiness, japonica rice starchpolysaccharide gel chewiness was lower. ${ }^{[23]}$ The increase in chewiness seen in Fig. 4C was consistent with gluten-free bread and pasta as a result of the EWPS concentration increase. ${ }^{[12,17]}$ In pasta, the link of disulfide bonds could be responsible for this effect through the thiol exchange between semolina proteins and EWPS when subjected to heat, while also establishing hydrophobic interactions. ${ }^{[12,24]}$ Disulfide bonds are formed through the gel formation of egg white ${ }^{[24]}$, possibly making disulfide bridges with gluten proteins in the pancake flour. ${ }^{[12]}$

\subsection{Fresh pancake pore size and SEM Images}

The pancake SEM image analysis allowed us to obtain a distribution of the pancake pore size between the different treatments. It was shown that the samples had a higher count for the pore size in the 1 to $3 \mathrm{~mm}$ diameter range. No significant differences were found between the treatments. Meaning the pore size count derived from the bubble size produced during the chemical leavening did not differ between the treatments. The leavening agents present in the pancake mix might influence the pore count since batter type cakes do not depend solely on egg foam for its leavening. ${ }^{[25]}$ Furthermore, the M200 samples network was reinforced due to what we assume is EWPS, shown as filaments limited to the surface of the bubbles or pores (red circles). Also, these results indicate that the higher thickness obtained in M200 treatment could be due to the network development consisting of protein filaments, seen to a lesser extent in P110. As a result, M200 could enable to entrap air incorporated during the process and cooking step, in a controlled and stable form.

Additionally, a pancake structure observed through confocal laser scanning microscopy (CLSM) showed a higher proportion of the gluten network around the disperse phase (air) in some areas. ${ }^{[26]}$ Such distribution is favorable for the 
network reinforcement provided by EWPS-gluten disulfide with M200 to confirm its distribution in the pancake matrix complemented with the analysis of the batter rheology, to understand how the protein and carbohydrate interactions influence its structure. Therefore, understanding how M200 is responsible for the improvement of pancake physical properties based on its network development.

\section{Discussion}

The use of chlorinated soft wheat flour in the bakery industry enables producers to obtain a product with high volume and even crumb in recipes using more sugar than flour. ${ }^{[9,27]}$ Nowadays, efforts to reduce sugar in bakery products in order to combat obesity, cardiovascular diseases, and type-2 diabetes compromise the quality of bakery products in which sugar has various functions. ${ }^{[28,29]}$ Furthermore, undesirable physical and texture properties in frozen bakery products have been observed. ${ }^{[11]}$ Studies showed egg white powder as an alternative due to its convenience and excellent foaming and binding properties. ${ }^{[2,12,17]}$

The present study showed the benefits of incorporating egg white powder into pancakes. M200 hydrophobic domains exposed to the aqueous phase enables it to adsorb rapidly at the air/water interface while stabilizing the generated air bubbles $^{[17]}$, helping in obtaining a thicker product when combined with whole egg as shown in Fig. 1A and 1C. In T2, both EWPS foaming properties might enable them to obtain a bridges around the pores. A similar study should be realized thicker product. This property is of importance as cakes batters are in part, foam with air incorporated during the whisked step, making the batter rise. ${ }^{[10]}$ Regarding hardness, Xie et al. showed water retained in pasta dough and the product was higher for M200 as the storage modulus ( $G^{\prime}$ and $\left.G^{\prime \prime}\right)$ increased when the dough was cooled from $90{ }^{\circ} \mathrm{C}$ to $40{ }^{\circ} \mathrm{C} \cdot{ }^{[12]} \mathrm{Gels}$ from alkaline egg white have shown a superior water holding capacity compared to low $\mathrm{pH}$ egg white. ${ }^{[30]}$ Therefore, a similar behavior could be attributed to pancake batter, although further studies should evaluate water activity and moisture content. A high specific volume is also associated with a low hardness in bakery products as gluten-free bread, also observed in our study on pancakes containing M200 with a high thickness. ${ }^{[31]}$

Egg white gelling characteristics are influenced by solutes

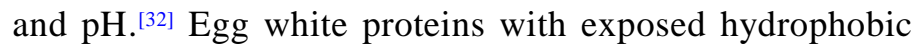
groups unfold to create a homogeneous network with high elasticity and cohesiveness. ${ }^{[30]}$ However, recent studies showed a decrease in hardness/gumminess/chewiness derived from egg white treated with $\mathrm{NaOH}$, attributed to a partial conformation unfolding of egg white proteins forming a weak strength elastic gel. ${ }^{[33]}$ These results coincide with the texture properties obtained in pancakes with M200. Therefore, the gelling mechanism could be different in the pancake matrix, arranged around the sides of the pores or bubble surface, as shown in Fig. 5. To evaluate consumers' preference, a
A
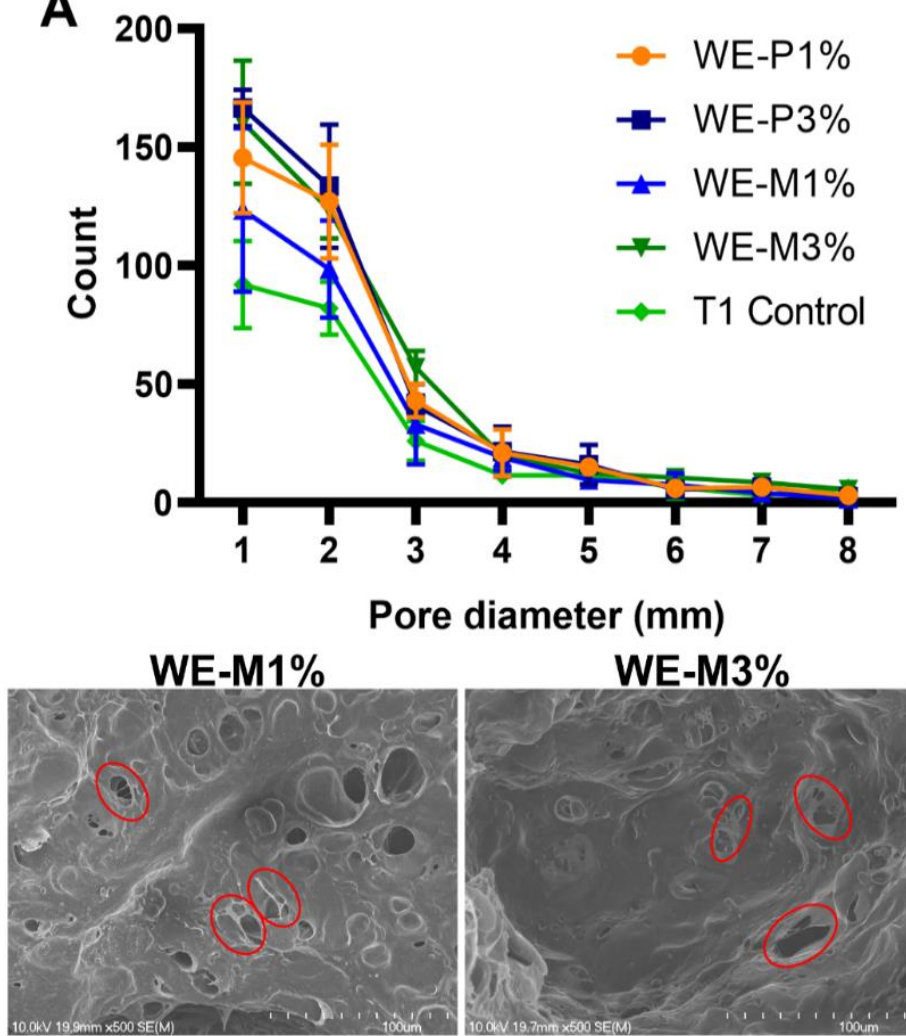

WE-M3\%

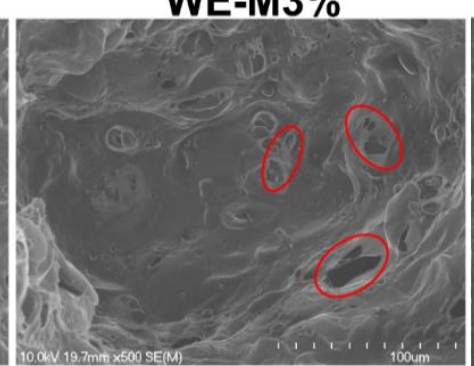

B
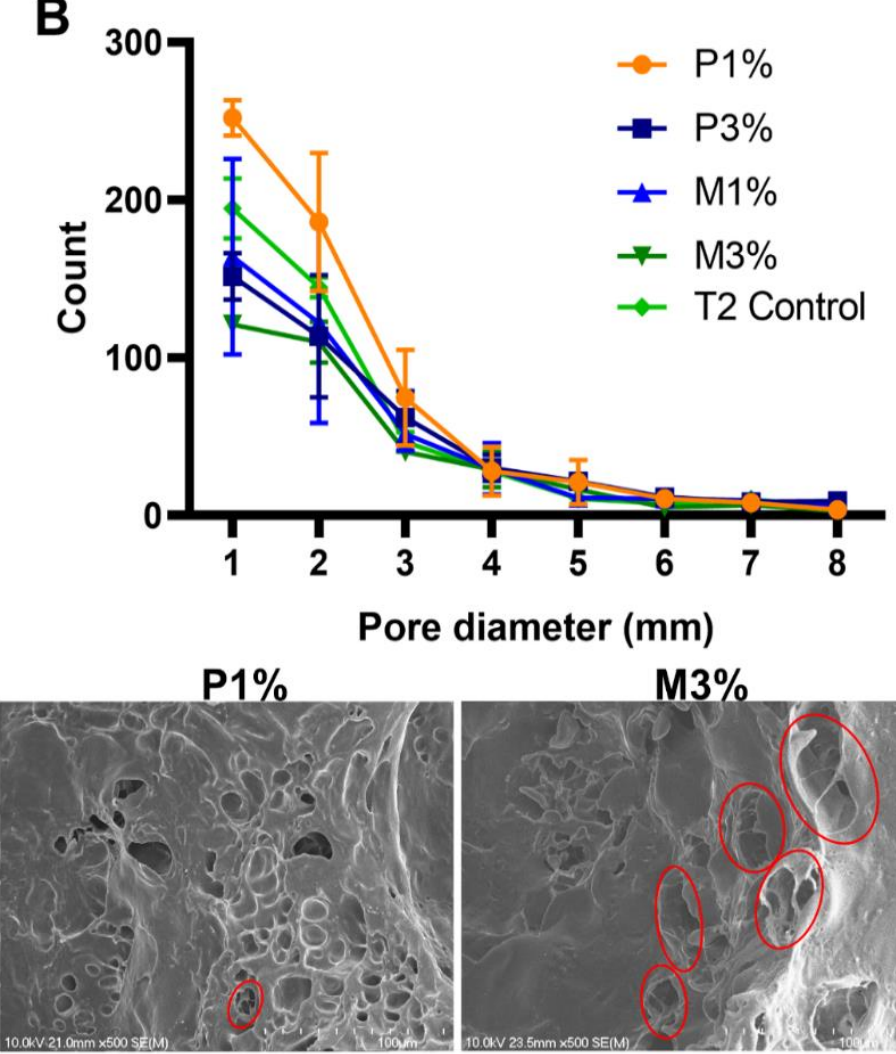

$\mathrm{M} 3 \%$

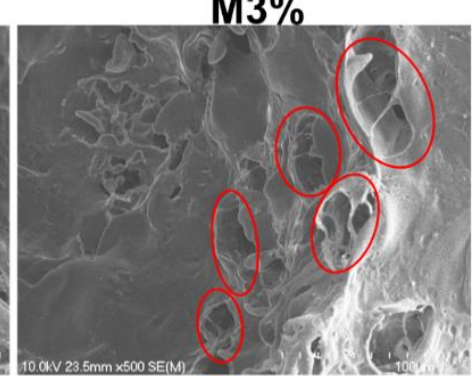

Fig. 5 Pore size distribution of treatment 1 (A), treatment 2 (B), and Scanning Electron Microscope (SEM) images of fresh pancakes. Numbers are mean \pm standard error of the mean $(n=2)$. 
preliminary sensory study using a hedonic analysis with untrained panelists $(n=23)$ was performed, taking into account the parameters as aroma, texture, flavor, dryness, and overall liking. No significant differences were observed between the $\mathrm{C} 1, \mathrm{WE}-\mathrm{M} 3 \%$, and WE-P3\% in all the parameters (Table S1), differing from the texture analysis results. Studies performing a TPA complemented with sensory evaluation in bread loaves observed a high correlation between the two methods on hardness evaluation with trained panelists. The trained panelists were able to detect differences in hardness between treatments. ${ }^{[34]}$ Therefore, further sensory studies on the pancakes with higher number of trained panelists are necessary to evalaute the improvement of the texture of pancakes prepared with EWPS.

\section{Conclusion}

To conclude, the present study shows the effect of EWPS on pancakes under fresh and frozen conditions. The formulation containing EWPS M200 achieved a higher thickness based on its superior foaming properties. The pancake's hardness and gumminess were lower in treatments containing M200 due to its documented water binding properties. Formulations without the whole egg found an increase in hardness proportional to EWPS content due to its gelling properties. The pancake chewiness was influenced by the EWPS ability to form disulfide bonds with gluten proteins, obtaining with M200 a pancake with less chewiness. No difference in pore size count was found between the treatments. However, the pancake network was possibly reinforced by M200, obtaining beneficial physical and textural properties in fresh and frozen pancakes.

Recommendation for future studies includes (i) a sensory evaluation with a trained panel with optimum number of participants to determine if the textural properties favor the consumer acceptability, (ii) a scale-up process to assess the manufacturing feasibility, (iii) a confocal microscopy study or similar to determine M200 distribution in the food matrix, and (iv) studying the freezing rate and EWPS percentage on improving frozen pancakes textural properties. This information could be of great value to the bakery industry seeking to reduce sugar content in their products, producing pancakes with improved physical and textural properties derived from low-cost incorporation of EWPS with the potential to increase consumer acceptability and consumption.

\section{Acknowledgments}

Kewpie Corporation financially supported this study. The authors are thankful to Henningsen Foods, Inc., which provided P110 and M200 EWPS. The authors are also grateful to Dr. You Zhou and Julia Russ from the University of Nebraska-Lincoln microscopy core for their guidance in the SEM use.

\section{Supporting information}

Not applicable.

\section{Conflicts of interest}

All contributing authors declare no conflicts of interest.

\section{References}

[1] Y. Mine, Trends Food Sci. Technol., 1995, 6, 225-232, doi: 10.1016/S0924-2244(00)89083-4.

[2] Y. Mine, Worlds. Poult. Sci. J., 2002, 58, 31-39, doi: 10.1079/WPS20020005.

[3] X. Wang, L. Gu, Y. Su, J. Li, Y. Yang and C. Chang, Food Hydrocoll., 2020, 101, 105530, doi: 10.1016/j.foodhyd.2019.105530.

[4] C. Chang, Y. Xu, M. Shi, Y. Su, X. Li, J. Li and Y. Yang, Food Hydrocoll., 2020, 99, 105333, doi: 10.1016/j.foodhyd.2019.105333.

[5] R. L. Barndt and R. N. Antenucci, in Low-Calorie Foods and Food Ingredients, ed. R. Khan, Springer Science and Business Media LLC, New York, 1993, pp. 106-137.

[6] E. Cho, J. E. Kim, B. K. Baik, J. B. Chun, H. Ko, C. Park and S. W. Cho, J. Food Sci. Technol., 2019, 56, 1349-1359, doi: 10.1007/s13197-019-03607-x.

[7] C. F. Morris and S. P. Rose, in Cereal Grain Quality, eds. R. J. Henry and P. S. Kettlewell, Springer Netherlands, Dordrecht, 1996, pp 3-54.

[8] S. M. Finnie, A. D. Bettge and C. F. Morris, Cereal Chem., 2006, 83, 684-691, doi: 10.1094/CC-83-0684.

[9] G. M. Bosmans, L. J. Peene, I. Van Haesendonck, K. Brijs and J. A. Delcour, Food Chem., 2019, 274, 434-443, doi: 10.1016/j.foodchem.2018.09.019.

[10] S. P. Cauvain and L. S. Young, in Bakery Food Manufacture and Quality, Wiley-Blackwell, Oxford, 2nd ed., 2009, pp. 111142.

[11] N. Jongsutjarittam and S. Charoenrein, Carbohydr. Polym., 2013, 97, 306-314, doi: 10.1016/j.carbpol.2013.04.087.

[12] L. Xie, N. Nishijima, Y. Oda, A. Handa, K. Majumder, C. Xu and Y. Zhang, Lwt, 2020, 122, 109031, doi: 10.1016/j.lwt.2020.109031.

[13] X. Duan, J. Li, Q. Zhang, T. Zhao, M. Li, X. Xu and X. Liu, Food Chem., 2017, 228, 243-248, doi: 10.1016/j.foodchem.2017.02.005.

[14] A. Kato, H. R. Ibrahim, H. Watanabe, K. Honma and K. Kobayashi, J. Agric. Food Chem., 1989, 37, 433-437, doi: 10.1021/jf00086a036.

[15] H. P. Song, B. Kim, J. H. Choe, S. Jung, K. S. Kim, D. H. Kim and C. Jo, Radiat. Phys. Chem., 2009, 78, 217-221, doi: 10.1016/j.radphyschem.2008.10.001.

[16] C. W. Pernell, P. J. Luck, E. A. Foegeding and C. R. Daubert, J. Food Sci., 2002, 67, 2945-2951, doi: 10.1111/j.13652621.2002.tb08843.x.

[17] A. Han, H. M. Romero, N. Nishijima, T. Ichimura, A. Handa, C. Xu and Y. Zhang, Food Hydrocoll., 2019, 87, 287-296, doi: 10.1016/j.foodhyd.2018.08.022.

[18] R. Crockett, P. Ie and Y. Vodovotz, Food Chem., 2011, 129, 84-91, doi: 10.1016/j.foodchem.2011.04.030.

[19] A. Farahnaky, R. Azizi, M. Majzoobi, G. Mesbahi and N. Maftoonazad, Innov. Food Sci. Emerg. Technol., 2013, 20, 173 
181, doi: 10.1016/j.ifset.2013.06.002.

[20] Y. Phimolsiripol, U. Siripatrawan and C. J. K. Henry, J. Food Eng., 2011, 105, 557-562, doi: 10.1016/j.jfoodeng.2011.03.022. [21] C. S. Brennan, M. Suter, T. Luethi, L. Matia-Merino and J. Qvortrup, Starch/Staerke, 2008, 60, 23-33, doi: 10.1002/star.200700670.

[22] P. A. Williams and G. O. Phillips, in Handbook of Hydrocolloids (Second Edition), eds. G. O. Phillips and P. A. Williams, Woodhead Publishing, Second Edi., 2009, pp. 252273.

[23] M. Huang, J. F. Kennedy, B. Li, X. Xu and B. J. Xie, Carbohydr. Polym., 2007, 69, 411-418, doi: 10.1016/j.carbpol.2006.12.025.

[24] S. Phongthai, S. D'Amico, R. Schoenlechner, W. Homthawornchoo and S. Rawdkuen, LWT - Food Sci. Technol., 2017, 80, 378-385, doi: 10.14416/j.ijast.2018.06.001.

[25] E. Wilderjans, A. Luyts, K. Brijs and J. A. Delcour, Trends Food Sci. Technol., 2013, 30, 6-15, doi; 10.1016/j.tifs.2013.01.001.

[26] S. Yamashita, K. Matsumiya, Y. Kogo, K. Takamatsu and Y. Matsumura, Food Hydrocoll., 2020, 100, 105432, doi: 10.1016/j.foodhyd.2019.105432.

[27] B. M. Gough, M. E. Whitehouse and C. T. Greenwood, $C R$ C Crit. Rev. Food Sci. Nutr., 1978, 10, 91-113, doi: 10.1080/10408397809527245.

[28] A. W. Sahin, E. Zannini, A. Coffey and E. K. Arendt, Food Res. Int., doi: 10.1016/j.foodres.2019.108583.

[29] R. A. Clemens, J. M. Jones, M. Kern, S. Y. Lee, E. J. Mayhew, J. L. Slavin and S. Zivanovic, Compr. Rev. Food Sci. Food Saf., 2016, 15, 433-470, doi: 10.1111/1541-4337.12194.

[30] K. Nyemb, C. Guérin-Dubiard, S. Pézennec, J. Jardin, V. Briard-Bion, C. Cauty, S. M. Rutherfurd, D. Dupont and F. Nau, Food Hydrocoll., 2016, 54, 315-327, doi; 10.1016/j.foodhyd.2015.10.011.

[31] C. Marco and C. M. Rosell, Eur. Food Res. Technol., 2008, 227, 1205-1213, doi: 10.1007/s00217-008-0838-6.

[32] M. Khemakhem, H. Attia and M. A. Ayadi, Food Hydrocoll., 2019, 87, 11-19, doi: 10.1016/j.foodhyd.2018.07.041.

[33] J. Li, Y. Zhang, Q. Fan, C. Teng, W. Xie, Y. Shi, Y. Su and Y. Yang, Food Chem., 2018, 250, 1-6, doi: 10.1016/j.foodchem.2018.01.031.

[34] P. M. Scheuer, M. Di Luccio, A. W. Zibetti, M. Z. de Miranda and A. de Francisco, J. Texture Stud., 2016, 47, 14-23, doi: $10.1111 /$ jtxs. 12155 .

\section{Author information}

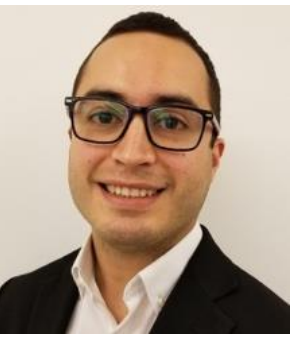

Emerson Nolasco was born in Tegucigalpa, Honduras. He completed his B.Sc. in Food Science and Technology in the Panamerican Agricultural School Zamorano, Honduras in 2015. Afterward, he worked on a palm oil refinery as a production supervisor for two years. He joined University of Nebraska-Lincoln in 2017 and obtained an M.S. degree in Food Science and Technology in 2019. Currently he is a PhD student in the University of NebraskaLincoln at the Department of Food science and technology.

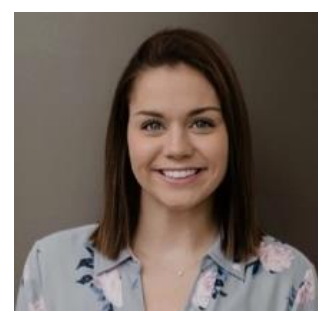

Emily Jundt was born in Lincoln, Nebraska, USA. She began her journey at the University of Nebraska-Lincoln in 2016 and aiming to graduate by fall 2020 with a major in food science.

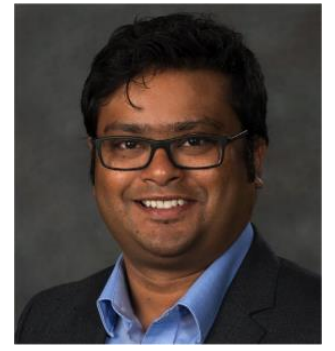

Kaustav Majumder is an Assistant Professor at the Department of Food Science, University of Nebraska, Lincoln, USA. He obtained his Ph.D. in Food Science and Technology from the University of Alberta, Canada in 2015, and did his Postdoctoral training at the University of Guelph, Canada. His research program at the University of Nebraska-Lincoln explores the beneficial health effect of food-derived bioactive proteins and peptides and evaluates their therapeutic potential for the treatment, prevention, and management of metabolic disorders.

Publisher's Note: Engineered Science Publisher remains neutral with regard to jurisdictional claims in published maps and institutional affiliations. 